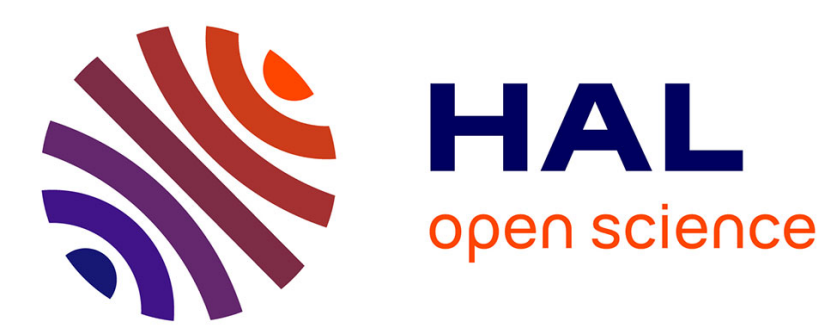

\title{
Study of Gear Surface Texture Using Mallat's Scattering Transform
}

\author{
W Sun, Stephane Chretien, R Hornby, P Cooper, R Frazer, J Zhang
}

\section{To cite this version:}

W Sun, Stephane Chretien, R Hornby, P Cooper, R Frazer, et al.. Study of Gear Surface Texture Using Mallat's Scattering Transform. International Conference on Advanced Mathematical and Computational Tools in Metrology and Testing (AMTCM 2017), Aug 2017, Strathclyde, United Kingdom. hal-01790302

\section{HAL Id: hal-01790302 \\ https://hal.science/hal-01790302}

Submitted on 11 May 2018

HAL is a multi-disciplinary open access archive for the deposit and dissemination of scientific research documents, whether they are published or not. The documents may come from teaching and research institutions in France or abroad, or from public or private research centers.
L'archive ouverte pluridisciplinaire HAL, est destinée au dépôt et à la diffusion de documents scientifiques de niveau recherche, publiés ou non, émanant des établissements d'enseignement et de recherche français ou étrangers, des laboratoires publics ou privés. 


\title{
Study of Gear Surface Texture Using Mallat's Scattering Transform
}

\author{
W. Sun *, S. Chrétien, R. Hornby, P. Cooper \\ National Physical Laboratory, Hampton Road, Teddington, TW11 OLW, UK \\ *E-mail: wenjuan.sun@npl.co.uk \\ R. Frazer, J. Zhang \\ Newcastle University, Newcastle upon Tyne, NE1 7RU, UK
}

\begin{abstract}
Gears are machine elements that transmit rotary motion and power by the successive engagements of teeth on their periphery ${ }^{1}$. Gears commonly in use include: spur gears; bevel gears; helical gears; internal gears and worm gears. They are widely used in automotive, aerospace, power generation and even medical applications. The manufacturing process of metal gears usually involves cutting, hobbing, shaving, milling, grinding and honing ${ }^{2}$. Although gears have been used for many years in industry, the evaluation of their surface texture and the relationship between surface parameters and surface functionality are not well understood. Conventional profile measurements and surface roughness parameters, such as $R a$ and $R q$, are still predominantly used in the industry. However, increasing investigations show that 2D profile measurements and data analyses cannot represent the surface condition due to nonuniformity of measured surfaces. In this study, we use a new mathematical tool for the characterisation of the surface irregularities, namely the scattering transform, recently introduced by S. Mallat ${ }^{3}$. This new transform is almost invariant to group actions such as rotation and translation of the image and has been successfully applied to machine learning problems such as classification. This approach is applied to the characterisation of gear surfaces. Results obtained on areal surface data based measurements from focus variation instruments and conventional tactile CMM are presented and discussed. ]
\end{abstract}

Keywords: Surface metrology; Scattering transform; Gaussian Mixture Models.

\section{Introduction}

Gears are a key component of power transmission systems, with applications spanning the automotive, aerospace, medical and power generation sectors, to name a few. A gear works by engaging its peripheral teeth with those of another gear, allowing rotary power to be transferred from one gear to the other gear. A system of gears can be arranged to modify the speed and direction of a rotation and torque to match the requirements for the efficient operation of the prime mover(electric motor, diesel engine etc.) with requirements of the 
driven load. This ensures the maximum operational efficiency and minimises manufacturing costs and environmental impact. The interaction between two gears is a combination of rolling and sliding. Different sections of gear teeth experience different meshing conditions, which influence the performance and the failure modes of the gear. The characterisation of the surface texture in the sliding direction root to tip for a driving gear or tip to root in a driven gear) is therefore of prime importance. The schematic of a gear tooth is shown in Figure 1.

Gears are commonly manufactured from case-hardened steel and are commonly produced by soft cutting followed by heat treatment and a hard-finishing process such as form grinding, honing or other processes to reduce the gear meshing surfaces roughness and form deviation. The root fillet regions also undergo grinding in some instances. The grinding process results in a grinding lay, illustrated in Figure 2, which is aligned approximately perpendicularly to the gear sliding direction. The lay is the direction of the predominant surface pattern. Lay usually derives from the actual production process used to manufacture the surface and results in directional striations across the surface. Figure 3 shows the common gear manufacturing and lay directions.

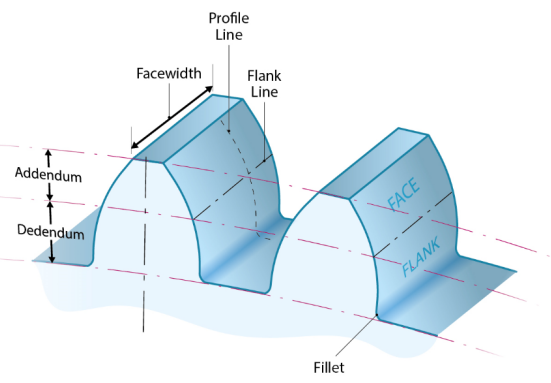

Fig. 1. Gear geometry vocabulary relevant to the measurements.

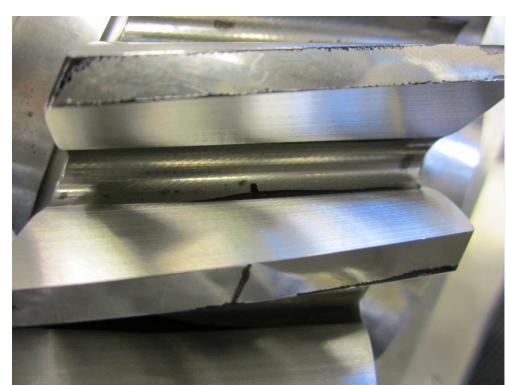

Fig. 2. Grinding lay from a gear form grinding machine 


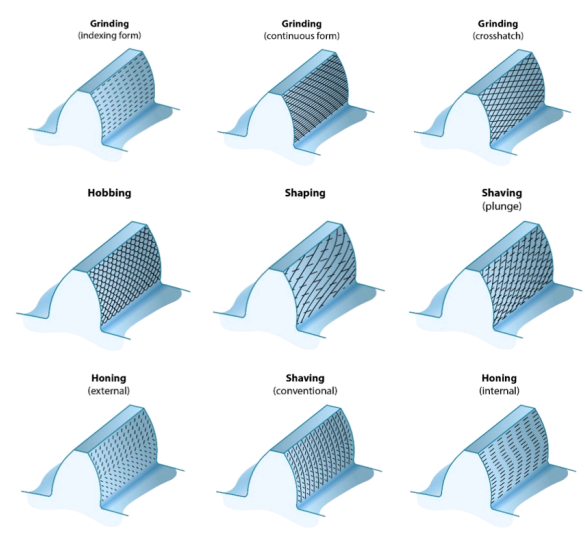

Fig. 3. Common gear manufacturing processes and lay directions. Reproduced from BS ISO TR 10064-4 ${ }^{4}$.

Among many gear manufacturing processes, grinding process is increasingly used. Grinding is an abrasive machining process that uses a grinding wheel to shape and finish components. The surface finish obtained through grinding can be significantly better than the surface finish obtained through other common processes, such as turning or milling. The process tends to produce repeatable form deviations on each gear tooth, but the grinding wheel can become blunt and its surface can be contaminated over time.

It is common to redress or re-cut the form of the grinding wheel after the grinding wheel has cut a specified number of teeth. Gear teeth ground after the grinding wheel has been redressed will likely have different surface deviations and cutting characteristics from those ground before it. Additional surface texture measurements are necessary to fully quantify the parameters of the gear if the grinding wheel has been redressed. Knowledge of the redressing process, and when it was performed during gear grinding, is required to develop an efficient and effective measurement strategy.

The characterisation of surface properties is essential in many industrial applications. A surface profile can be decomposed into three components: primary (often defined in terms of geometric element such as a line or a circular arc), the waviness and roughness. Among them, surface roughness is the important components that affects the friction, durability and also the failure mode of components. The common metrics in metrology are the roughness $R$ parameters ${ }^{15}$. The procedure of surface roughness analyses includes filtering of surface irregularities (noise etc.), removing surface form and surface waviness. The resulting surface roughness can then be parameterised using roughness parameters such as $R a, R z, R s q$, and etc. However, the drawback of using surface profile is that the information taken is dependant of the orientation of the scan relative to the surface lay, see examples in Figure 4.

In recent years, international standards ISO 25178 introduced $S$ parameters for surface texture analyses. The fundamental theory behind $R$ and $S$ parameters is the same, but $S$ parameters have advantages over conventional $R$ parameters as $S$ parameters take into the consideration of areal data rather 
than single profile. However, for both sets of parameters, it can be difficult to correlate the measured values of the parameters with the surface functionality. In fact, different manufacturing processes may generate different surface components in different wavelength regimes and these affect the function of the surface differently. Filtering surface profiles to finer descriptors of the subtle variations in the process can provide a better idea of the surface. The process can be performed using Wavelet analysis ${ }^{3}$. Previous researchers used wavelets for texture analysis and a very interesting trend emerged that progressively refined the wavelet approach to metrology ${ }^{5}$. While very efficient for some applications, the wavelet transform cannot aggregate (local) spatial patterns in a natural fashion and we need to turn to new techniques which can help extract the main metrological spatial features of surfaces by building relevant invariants under local translations and rotations. This can be achieved using Mallat's scattering transform.

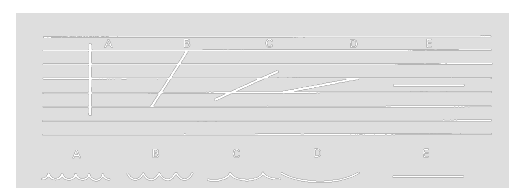

Fig. 4. The effect of measuring in different directions to the surface lay, courtesy of Taylor Hobson Ltd. Note that the direction of lay runs across the page.

In this paper, Mallat's Scattering Transform is used as an promising new tool for surface characterisation and metrology. Mallat scattering transform is a new technique enjoying fruitful connections with deep learning ${ }^{3}$ and a sound mathematical theory. The advantage of the scattering transform over traditional techniques is that it leverages the intrinsic multi-scale analysis of wavelet analysis while remaining almost invariant to certain local group actions. In this paper, we show via numerical experiments how these new metrics reflect the properties of the surface. This study is a step towards a wider use of the scattering transform in metrology.

\section{Mallat's scattering transform}

In this section, we present the Scattering Transform proposed by Mallat ${ }^{3}$ and describe its construction and main properties. The scattering transform provides a compact description of images or surfaces that are invariant with respect to the action of certain local transformations. The application of the scattering transform to metrology is a promising avenue for a precise multiscale analysis of surfaces and a new approach to the study of roughness and of surface description in general.

The scattering transform builds up on Wavelet theory. Wavelet analysis have been widely used for texture analysis and application to metrology ${ }^{6}, 7$. Wavelets are able to efficiently delineate roughness from waviness, a difficult but essential problem in surface metrology.

One of the main pitfalls of wavelets is that they are not so much invariant to group actions such as rotations or translations. In order to overcome this 
issue the scattering transform ${ }^{3}$ was recently proposed so as to represent images and to capture their main features for the task of classification in machine learning. Moreover, one of the main advantages of the scattering transform over competitors such as deep neural networks is that it is underpinned by a sound theory ${ }^{3}$.

The scattering transform provides an architecture that computes interesting features that are invariant to local transformations. It consists of a succession of linear filters and 'pooling' non-linearities. The same type of architecture is deployed in e.g. deep neural networks with the main difference that the weights have to be computed from the data, a task that is not known to be efficiently achievable despite interesting recent advances ${ }^{8},{ }^{9},{ }^{10}$. Mallat's scattering transform proposes stable and informative affine invariant representations which can quickly be obtained via a scattering operator defined on the translation, rotation and scaling groups. It is implemented by a deep convolution network with wavelets filters and modulus non-linearities, hence allowing interesting connections with deep neural networks.

\subsection{Invariants}

Building representations that are invariant to certain actions is important in many applications. In the metrological applications we want to address, the features that should be retrieved from the analysis should not be impacted by e.g. small translations, small deformations or small rotations. This is what we can achieve using the scattering transform.

The scattering transform can be described as a cascade of wavelet coefficient computations composed with the non-linear operation of taking the modulus and averaging, all this being repeated at several scales.

As is usual in wavelet analysis, one defines a scaling function $\phi$ whose translates define the $0^{t h}$ resolution space $V_{0}$ and $\phi_{J}(\cdot)=\phi\left(2^{J} \cdot\right)$. Let $f \in L^{2}(\mathbb{R})$, the Hilbert space of squared integrable functions ${ }^{11}$. Then, the convolutions of $f$ with the scaling function $\phi_{J}$, which has characteristic support size of order $2^{J}$

$$
f \mapsto f * \phi_{J}
$$

where $*$ denote the discrete convolution operator ${ }^{11}$. This convolution filters out higher frequencies from the signal and gives the average behaviour of the function. This average is not much perturbed by small translations, or in other words,

$$
f * \phi_{J} \approx f(\cdot-\tau) * \phi_{J}
$$

when $|\tau| \ll 2^{J}$.

\subsection{The scattering mechanism and higher frequency invariants}

In order to refine the analysis by incorporating higher frequencies, one introduces the wavelet functions $\psi_{j}, j=1, \ldots, J$ which are obtained in such a way that their translates form a orthogonal basis of $V_{j} \backslash V_{j-1}$. where $A \backslash B$ here and hereafter will denote the orthogonal complement of vector space $B$ inside vector space $A$. One proceeds to computing the vector

$$
\left|f * \psi_{1}\right| * \phi_{J}, \quad\left|f * \psi_{2}\right| * \phi_{J}, \quad \ldots
$$


which contains information about the higher frequencies contained in $f$. Again, the averaging is crucial at this point because it helps robustifying the transform against translation-type perturbations. On the other hand, the high frequencies are again lost by this averaging procedure. The scattering transform then consists of the following list of coefficients:

$$
\begin{aligned}
& || f * \psi_{1}\left|* \psi_{1}\right| * \phi_{J},|| f * \psi_{2}\left|* \psi_{1}\right| * \phi_{J}, \cdots \\
& || f * \psi_{1}\left|* \psi_{2}\right| * \phi_{J},|| f * \psi_{2}\left|* \psi_{2}\right| * \phi_{J}, \cdots \\
& || f * \psi_{1}\left|* \psi_{3}\right| * \phi_{J},|| f * \psi_{2}\left|* \psi_{3}\right| * \phi_{J}, \cdots
\end{aligned}
$$

\subsection{Sparsity of scattering coefficients}

One fundamental feature of the wavelet expansion is that it sparsifies functions from many standard function spaces such as Besov spaces, piecewise differentiable functions, functions of Bounded Variation, etc; see ${ }^{11}$ for more details. In other words, most coefficients in the wavelet expansion will be negligible, allowing for a sparse representation of the function under study. These properties mainly stem from the fact that wavelet bases are unconditional bases for these spaces and unconditionality implies providing nearly sparse (also called compressible) expansions as proved in ${ }^{12}$. The study of the decay of the scattering coefficients was completed in ${ }^{13}$. In that paper, it is proved that most coefficients in the scattering decomposition are small for functions in $L^{2}(\mathbb{R})$. More precisely, we have

$$
\begin{aligned}
& \sum_{m \geq n}\left\|S_{m}[f]\right\|_{2}^{2} \\
& \leq \int_{\mathbb{R}}|\hat{f}(\omega)|^{2}\left(1-\exp \left(-\frac{\omega}{r a^{n}}\right)\right) d \omega
\end{aligned}
$$

and, as easily seen, the function

$$
\omega \rightarrow 1-\exp \left(-\frac{\omega}{r a^{n}}\right)
$$

is a high pass filter with a bandwidth proportional to $r a^{n}$.

\subsection{Going from $1 D$ to $2 D$}

Since the Scattering Transform can be applied to images and is invariant to rotations ${ }^{14}$, the material's features will be detected independent of the direction. This might represent a great advantage over the previous approach in many situations.

Note that the 1D Scattering Transform can still be used in the case one wants to compare with the usual $1 \mathrm{D}$ roughness criteria.

\section{Computational experiments}

Three gear tooth surface replica, provided by Design Unit at University of Newcastle, were studied. One gear tooth was finished by grinding process (as 


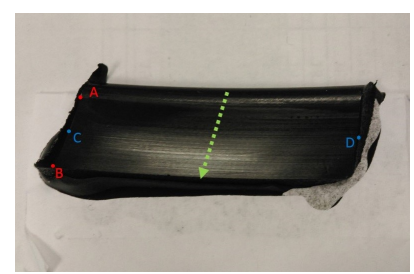

Fig. 5. Top down view of Microset 202 black replica with points used for alignment highlighted.

ground), one was used for short period, little load (run in), and the other sample was used for a long time, significant load, high speed (test 1).

Figure 6 below shows the scan results of these three components using the focus variation system.

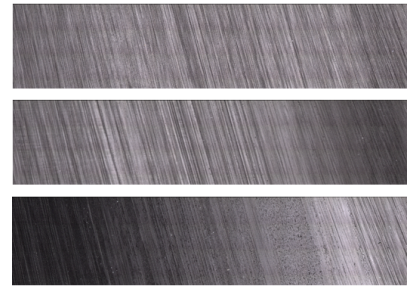

Fig. 6. From top to bottom, as grand, after run in and after test 1.

\subsection{Results}

One example of surface we have to analyse is shown in Figure 7.

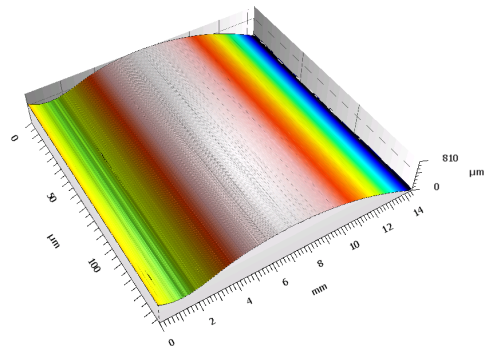

Fig. 7. Surface image from the "test 1" set.

The sparsity of the scattering transform can be observed in Figure 8. This figure shows the scattering coefficients sorted in absolute value. The cluster 


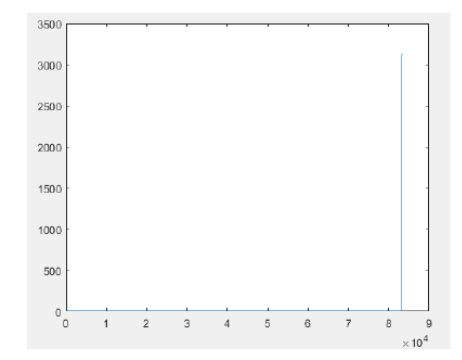

Fig. 8. Scattering coefficients of the previous image sorted by increasing absolute value. The $y$-axis represents the magnitude of the Scattering Transform coefficients. In particular, this figure shows an example of extreme sparsity of the Scattering Transform for our data set.

containing the $5 \%$ largest coefficients of the scattering transform are displayed in Figure 9 below. The support is the vector which containts the index of the

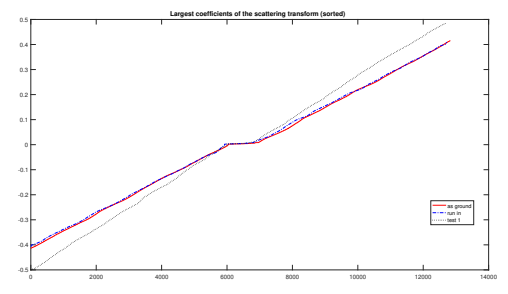

Fig. 9. The 5\% largest coefficients sorted in increasing order. The $y$ axis represents the coefficient's magnitude.

components displayed in Figure 9 above. The first difference of the support is the vector of all successive differences between consecutive values of the support. The second difference of the support, i.e. the first difference of the first difference of the support, is shown in Figure 10 below. Figure 11 shows a zoomed version of the middle part of Figure 10.

It can be seen from the previous plots that the scattering transform efficiently compresses the information contained in the surface figures. Only a few large coefficients have significant magnitude. Figure 9 and Figure 11 show that the information contained in the scattering coefficient sequence contains very interesting information. Firstly, the sorted magnitude grows linearly in Figure 9, which is a very interesting feature. Next, the second difference of the support is spiky (sparse). Moreover, Figure 10 shows that the sample as ground and run in have very similar scattering coefficients whereas the sample test 1 has a very different slope from the two other samples. This results seem correspond well to the physical difference between these three samples, where the sample test 1 is rough comparing to the other two samples. On the other hand, the support of the three samples are very different as shown in Figure 


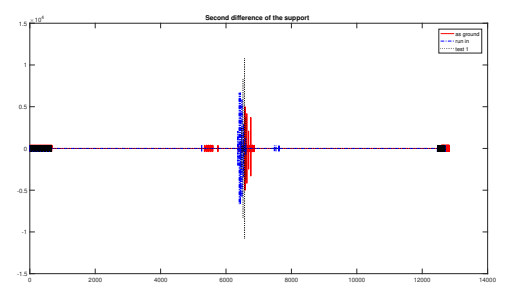

Fig. 10. The second difference of the support of the $5 \%$ largest coefficients.

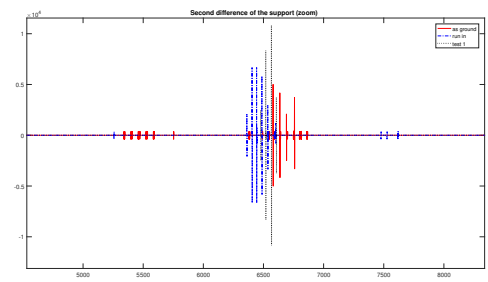

Fig. 11. Zoom into the second difference of the support of the $5 \%$ largest coefficients.

11. In particular, the second difference of the support of as ground and run in can be easily distinguished: the 'two-dashed' coefficients are larger than the 'solid' ones. Moreover, the blue coefficients are sparser than the red ones.

\section{Conclusion and future work}

The goal of the present paper was to introduce the scattering transform as a tool for the study of surface metrology. The preliminary study shows interesting results when applying this method to gear surfaces. The scattering transform is a first step to produce invariant features (the transform coefficients) that can be used to classify or predict the functionality the surface. In order to fully understand the method, the future research is to further investigate the metrological interpretation of the coefficients of Mallat's scattering transform, and in particular:

- the distribution of the magnitude of the largest coefficients

- the position of the largest coefficients in the sequence.

\section{Acknowledgements}

The project is jointly funded by the European Metrology Research Programme and the National Measurement System Programme for Engineering \& Flow Metrology. We are grateful to Prof Alistair Forbes for comments on an early draft of this paper. 


\section{References}

1. J. R. Davis, Gear materials, properties, and manufacture (ASM International, United States, 2005)

2. G. Goch, Gear metrology CIRP Ann-Manuf Techn. 52 (2) 659-95, (2003)

3. S. Mallat, Group invariant scattering Communications on Pure and Applied Mathematics 65 (10) 1331-98, (2012)

4. BS ISO/TR 10064-4:1998 Code of inspection practice Part 4: Recommendations relative to surface texture and tooth contact pattern checking, (1998)

5. S. H. Bhandari and S. M. Deshpande, Feature extraction for surface classification - An approach with wavelets. International Journal of Computer and Information Science and Engineering 1.4: 215-219 (2007).

6. X. Jiang and L. Blunt, Third generation wavelet for the extraction of morphological features from micro and nano scalar surfaces. Wear, 257(12), 1235-1240 (2004).

7. R. X. Gao, Robert and Y. Ruqiang, Wavelets: Theory and applications for manufacturing. Springer Science \& Business Media, (2010).

8. P. Hand and V. Voroninski, Global Guarantees for Enforcing Deep Generative Priors by Empirical Risk. arXiv preprint arXiv:1705.07576 (2017).

9. Q. Nguyen and M. Hein, The loss surface of deep and wide neural networks, arXiv preprint arXiv:1704.08045 (2017).

10. M. Soltanolkotabi, A. Javanmard and J. D. Lee, Theoretical insights into the optimization landscape of over-parameterized shallow neural networks, arXiv preprint arXiv:1707.04926 (2017).

11. S. Mallat, A wavelet tour of signal processing. Academic press (1999).

12. D. Donoho, Unconditional bases are optimal bases for data compression and for statistical estimation." Applied and computational harmonic analysis 1.1 (1993): 100115.

13. I. Waldspurger, Exponential decay of scattering coefficients, arXiv preprint arXiv:1605.07464 (2016).

14. L. Sifre and S. Mallat, Rotation, scaling and deformation invariant scattering for texture discrimination, in Proceedings of the IEEE conference on computer vision and pattern recognition, pp. 1233-1240 (2013).

15. BS EN ISO 4287:1998+A1:2009 Geometrical product specification (GPS) -Surface texture: Profile method - Terms, definitions and surface texture parameters, 1998 